HStud 27 (2013)1, 3-14

DOI: 10.1556/HStud.27.2013.1.1

\title{
LES ACTIVITÉS SECRÈTES ET JOURNALISTIQUES DU COMTE JULES ANDRÁSSY LE JEUNE EN SUISSE ET SES CONCEPTIONS DE L'APRÈS-GUERRE
}

\author{
ZOLTÁN BÉCSI \\ Institut européen de l'Université de Genève \\ Genève, Suisse \\ E-mail: zbecsi@gmail.com
}

\begin{abstract}
Ayant fuit la Hongrie pour un exil de huit mois en Suisse, Jules Andrássy retrouve les milieux contre-révolutionnaires en exil ainsi que le journaliste Félix Vályi pour œuvrer, par un travail de propagande, à la chute de la Commune en Hongrie, au retour du roi en exil et au combat pour l'intégrité territoriale de la Hongrie. Pendant son séjour, Andrássy écrit deux articles dans la Revue politique internationale, dirigée à Lausanne par Vályi. Ses articles dévoilent ses conceptions d'après-guerre sur l'avenir de l'Europe centrale et de la Hongrie. Il espère ainsi influencer la politique des vainqueurs de la Grande Guerre dans cette région.
\end{abstract}

Mots-clefs : Jules Andrássy, Félix Vályi, Suisse, France, légitimisme, GrandeBretagne, Entente, Quadruple Entente, renseignement, fédéralisme, confédéralisme, ABC (comité anti-bolchevique)

Nous allons présenter ici les destins croisés, en 1919, d'un homme politique hongrois et d'une revue de langue française, le comte Jules (Gyula) Andrássy le jeune et la Revue politique internationale, fondée par Félix Vályi et publiée entre 1914 et 1920. Nous nous limiterons aux contributions d'Andrássy, car une analyse plus générale de la revue et de son fondateur dépasserait le cadre d'un simple article. D'autre part, nous nous concentrerons sur 1919, année qu'Andrássy passa pour la plus grande partie en Suisse.

Avant de procéder à l'analyse des articles d'Andrássy, nous allons tout d'abord décrire les circonstances de son séjour en Suisse et de sa collaboration intense avec Vályi, qui prêta de bonne grâce les pages de sa revue pour faire de celle-ci l'instrument momentané du message d'Andrássy le jeune au monde et plus particulièrement aux hommes politiques de l'Entente. 


\section{Deux hongrois en Suisse : Félix Vályi et Jules Andrássy le jeune}

Au début du siècle, Félix Vályi était le correspondant parisien du Magyar Szó, organe des protestants libéraux hongrois publié entre 1900 et 1914. Ce quotidien s'était d'abord illustré comme le défenseur de l'esprit de la Réforme, puis comme celui d'une voie radicale et hongroise. ${ }^{1}$ En 1914, Vályi lança sa propre revue qu'il baptisa Revue politique internationale, dont il publia le premier numéro à Paris en janvier. Défendant une ligne internationale, objective et impartiale, scientifique et non polémique, la rédaction affirmait vouloir offrir à toutes les nationalités «1'hospitalité française d'une tribune libre ». ${ }^{2}$ Vályi parvint à obtenir la collaboration de personnalités françaises comme Aristide Briand, Paul Deschanel, Louis Eisenmann (historien connu pour ses sympathies slaves, plutôt qu' hongroises, qui allait soutenir la cause tchécoslovaque pendant la guerre), Emile Durkheim, Georges Sorel..., mais aussi des personnalités d'autres pays, comme l'ancien président du Conseil italien, Luigi Luzzatti, l'orientaliste britannique, Edward G. Browne, ou le sociologue et économiste allemand, Franz Oppenheimer, ainsi que le comte Albert Apponyi et bien sûr notre protagoniste, Jules Andrássy le jeune. Le secrétariat de rédaction de la revue fut confié au journaliste puis homme politique, André François-Poncet, qui allait être envoyé en 1917 à l'Ambassade de France à Berne, pour y accomplir une mission de renseignement. Après la guerre, François-Poncet allait accéder à une large notoriété, culminant avec l'élection à l'académie française en $1952 .^{3}$ C'était donc des gens de qualité qui se rassemblaient autour du berceau de la revue de Vályi, le niveau des publications en est d'ailleurs le reflet.

Après le déclanchement de la Grande Guerre, Vályi fut obligé de déplacer sa rédaction en territoire neutre, en Suisse et plus précisément dans le centre de Lausanne, Place Saint-François. Dès lors, André François-Poncet cessa d'être membre de la rédaction, mais il continua à collaborer à la revue. Dans un avis aux lecteurs, Vályi expliquait que sa revue devait rester « un organe d'études où les esprits du monde entier, sans distinction de nationalités, peuvent se mesurer dans une discussion loyale. ${ }^{4}$

Jules Andrássy le jeune, quant à lui, était un aristocrate, propriétaire foncier et homme politique, tout comme son père (dit l'aîné) qui avait été l'un des principaux artisans du compromis austro-hongrois en tant que président du conseil hongrois en 1867, avant de devenir Ministre des Affaires étrangères de François-Joseph. Jules Andrássy le jeune (plus loin, Jules Andrássy) était devenu député en 1885, à l'âge de vingt-cinq ans, puis secrétaire d'Etat (en 1892) et enfin ministre de Sa Majesté en 1894, c'est-à-dire ministre hongrois résidant à Vienne auprès du monarque. Il s'était par la suite consacré à la recherche scientifique (les études politiques) en devenant membre de l'Académie des Sciences hongroise. En 1906, il était revenu à la pratique politique en tant que ministre de l'Intérieur du 
gouvernement libéral de Sándor Wekerle. Le 25 octobre 1918, il avait été nommé par l'empereur-roi Charles ministre des Affaires étrangères, débarquant à Vienne dans la capitale d'un empire au bord de l'écroulement. Les révolutions, à Budapest comme à Vienne, l'avaient obligé à démissionner le 31 octobre 1918, quelques jours avant l'armistice. Suite à cela, il rentra dans sa propriété de Dénesfa, près de Sopron. Mais dès le mois de janvier 1919, il prit la route de l'exil vers la Suisse. ${ }^{5}$

Jules Andrássy se considérait alors comme investi d'une mission décisive, celle de sauver ce qui pouvait encore l'être de la monarchie des Habsbourg. Il ne considérait pas comme incompatibles la défense des intérêts de la dynastie et celle des intérêts du royaume de Hongrie. Dans le passé, les deux causes avaient souvent divergé ; le propre père d'Andrássy s'était lui-même révolté contre l'absolutisme des Habsbourg, mais il avait été, plus tard, l'un des principaux artisans du compromis austro-hongrois, avec le soutien et grâce à l'influence de l'impératrice Elisabeth d'Autriche. Il est donc, somme toute, peu surprenant que le jeune Andrássy souhaitât préserver l'héritage pour lequel son père avait si longuement travaillé et dont le résultat avait été cinquante années de paix de prospérité pour sa patrie, la Hongrie. De Suisse, pays neutre frontalier avec la France, il allait avoir le loisir de mettre en branle une propagande secrète en vue de la défense des intérêts de sa patrie. La revue de Vályi allait s'avérer un instrumental crucial à cet effet.

\section{Les activités des légitimistes en exil}

Andrássy résida en Suisse, de janvier à août 1919, exil pendant lequel eurent lieu les 133 jours de la Commune en Hongrie (du 21 mars au $1^{\text {er }}$ août 1919). Le 15 août, il rentra en Hongrie pour y continuer le combat politique en faveur du retour à la monarchie légitime et à l'intégrité territoriale de la Hongrie. ${ }^{6}$

$\mathrm{Au}$ cours de la même période, des hommes politiques hongrois qui avaient également fuit la Commune créèrent à Vienne l'ABC (Le Comité antibolchevique). Mais peu de légitimistes, hongrois comme autrichiens, croyaient la restauration de la monarchie possible en Autriche allemande. Dès lors, ils se concentrèrent sur la mise en échec de la Commune hongroise et sur la restauration, au plus vite, de l'état de droit et de la monarchie parlementaire en Hongrie grâce au retour du monarque légitime, Charles IV de Habsbourg, dans ses fonctions sur le trône de Saint Etienne ; fonctions royales qu'il avait suspendues le 13 novembre 1918 à la demande du Conseil National hongrois, présidé par le comte Mihály Károlyi, pour un laps de temps indéfini. 
À l'ABC de Vienne, toutes les nuances de la droite étaient représentées, auxquelles s'ajoutaient des sociaux-démocrates comme Ernő Garami, eux aussi impliqués dans les efforts entrepris pour renverser la Commune.

En Suisse, Andrássy faisait parti d'un petit groupe de légitimistes hongrois qui étaient, eux aussi, proche de l'ABC. ${ }^{7}$ Les membres prééminents du groupe étaient le prince Lajos Windischgraetz et Vilmos Vázsonyi. Pendant la guerre, Lajos Windischgraetz avait été ministre de l'alimentation. Restant un légitimiste convaincu, il allait continuer à défendre les droits des Habsbourg après la guerre. Quant à Vilmos Vázsonyi, c'était un avocat et homme politique libéral démocrate d'origine juive, ancien Ministre de la Justice et fondateur du Cercle démocratique (1894). Vázsonyi s'était en particulier fait connaître pour son combat en faveur de l'admission du Judaïsme comme religion d'Etat en Hongrie en $1895 .^{8}$

Après avoir rendu visite à leur souverain en exil à Prangins, près de Nyon (Canton de Vaud), ces quelques hommes se mirent au travail en vue de constituer une propagande légitimiste en Suisse tout en maintenant le contact avec l'ABC de Vienne. ${ }^{9}$ Le soutien financier d'un riche industriel autrichien résidant à Lucerne, le baron Andreas Veitsberger, leur permit d'établir un organe de presse baptisé Agence centrale. ${ }^{10}$ De plus, Félix Vályi mettait sa revue à leur disposition comme moyen d'influence de l'opinion publique, surtout française, dans le sens des intérêts de la Hongrie. Et enfin, ils étaient en contact avec le responsable du bureau de presse de l'Ambassade de France à Berne, Emil Haguenin, dont Andrássy avait déjà fait la connaissance pendant la guerre par le truchement de Vályi. Grâce à Haguenin, Andrássy avait accès aux cercles élevés du monde politique français. ${ }^{11}$

Les membres actifs de l'Agence étaient Andrássy, Windischgraetz, Vályi et un certain Harry Schmidt, de Genève, collaborateur de l'Intelligenz Blatt de Berne qui bénéficiait d'un passeport diplomatique suisse et faisait office d'agent de liaison entre les légitimistes de Hongrie et ceux qui résidaient en Suisse. ${ }^{12}$

L'agenda politique de l'Agence centrale fut très vite démasqué par les diplomates anglais de Berne : le Ministre de Sa Majesté britannique à Berne, Rumbold, affirmait ainsi en mars 1919 que le jeu de l'Agence était d'exagérer le danger bolchevique en Hongrie et en Autriche. ${ }^{13}$ Le proche avenir allait au contraire révéler que les légitimistes n'avaient guère exagéré la menace, puisque c'est dès la fin de ce même mois de mars que la République des Conseils allait être proclamée à Budapest. Il semble bien que Rumbold avait omis de souligner le plus important : l'Agence centrale avait pour but d'informer les media occidentaux de la vraie situation dans la région et de maintenir le contact avec les partisans du roi dans les différents états successeurs de l'Autriche-Hongrie, tout en influençant la presse internationale dans le sens de la nécessité de confédérer l'espace danubien. ${ }^{14}$ 
Le modus operandi de l'Agence était de s'appuyer sur une société d'import-export danoise qui avait créé une agence télégraphique économique possédant déjà, selon certaines sources, une filiale dans toutes les villes européennes d'importance, le réseau de bureaux à travers l'Europe serait à même de relayer des nouvelles sur la situation économique des pays de la région danubienne. Le réseau servirait aussi à maintenir le contact avec les milieux légitimistes de la région. En un mois, l'agence eut des filiales à Prague, Belgrade, Budapest, Londres et Paris. Toutes ces filiales étaient elles-mêmes en relation avec les principaux journaux de leurs pays respectifs et diffusaient des informations économiques afin de favoriser l'union économique des Etats danubiens. ${ }^{15}$

Nous pouvons déjà tirer certaines conclusions sur le profil des légitimistes de l'ABC, notamment sur ceux de la petite équipe de Suisse. Sociologiquement variés, ils étaient tout de même majoritairement d'origine aristocratique. Fait intéressant : quelle que fut leur origine sociale, les légitimistes que nous avons mentionnés étaient plutôt des libéraux. À la différence des royalistes allemands - ceux de l'entourage du Maréchal Ludendorff, qui se situaient à l'extrême droite - les légitimistes hongrois étaient à forte prédominance libérale démocratique ou conservatrice libérale.

La présence de Félix Vályi dans ce groupe est quelque peu surprenante, car il avait été le correspondant à Paris d'un journal hongrois radical et assez frondeur, pour lequel il avait fait des entretiens avec des hommes politiques français engagés dans l'anticléricalisme, comme Clemenceau qui avait fait l'apologie de la séparation de la Hongrie de l'Autriche des Habsbourg. ${ }^{16}$ Toutefois, l'évolution de la situation de la Hongrie après la guerre peut expliquer un rapprochement de Vályi avec le légitimisme. Le roi légitime de Hongrie pouvait en effet représenter pour les personnes de toutes sensibilités politiques un élément rassembleur capable de contribuer à la défense de l'intégrité territoriale de la Hongrie, mise à mal depuis 1918 ; le roi était au dessus de la mêlée des partis politiques et des nationalités (Slovaques, Croates, Roumains...). Ayant quitté l'Autriche-Hongrie en 1918, le roi Charles n'était pas tenu, croyait-on, par les engagements pris par les États successeurs nationaux envers l'Entente (surtout après la signature du traité de paix de Trianon, le 4 juin 1920, dans le cas de la Hongrie). Son retour pouvait donc avoir un effet centripète et permettre à la Hongrie de Saint Etienne de survivre et de retrouver ses territoires occupés. Une Hongrie nouvelle (sans continuité avec le passé), nationale et républicaine avait, en revanche, moins d'arguments légitimes pour justifier le retour de son intégrité d'avant 1918. À l’opposé, les républicains zélés, comme Mihály Károlyi, croyaient que la rupture avec la Double monarchie, qu'ils chargeaient de la responsabilité de la guerre, allait permettre de sauver l'intégrité territoriale. En fin de compte, aucune des deux approches n'allaient porter des fruits. 


\section{Les Articles d'Andrássy à la Revue politique internationale}

Outre ses activités de diplomatie secrète au service des Habsbourg et de la Hongrie, Jules Andrássy écrivit deux articles sur le futur de sa patrie dans la Revue politique internationale au cours de son exil en Suisse. Les circonstances de sa rencontre avec Vályi ne sont pas connues, mais Andrássy avait déjà publié trois articles dans la revue pendant la guerre. Même si nous concentrerons notre attention sur les articles d'après-guerre, il est intéressant de jeter un coup d'œil sur l'article publié en novembre 1916, intitulé Le problème de la paix, ${ }^{17}$ car il se projette déjà dans l'après-guerre.

L'article commence par un puissant appel en faveur des négociations de paix associé à la critique de l'Entente, surtout de la France, pour son rejet de la proposition allemande d'engager des discussions. ${ }^{18}$ Cet article contribua probablement au démarrage des négociations secrètes de paix entre la France et l'Allemagne, qui se déroulèrent en Suisse en hiver $1916-1917 .{ }^{19}$ Andrássy y esquisse déjà les conséquences de la victoire de la Quadruple Entente ${ }^{20}$ et croit que le partage de l'Europe et du proche et moyen Orient se fera entre la Grande Bretagne et la Russie. Il se trompait, car la révolution allait mettre la Russie hors jeux pour quelques temps. En revanche, Andrássy est pertinent lorsqu'il parle de l'Allemagne comme d'un danger nouveau, si la Quadruple Entente s'engage à fond pour la victoire totale : pour exprimer cette idée, il emploie l'expression de « revanche des peuples vaincus ». ${ }^{21}$ Tout d'abord, il écrit qu'il s'agit d'un peuple de quatre-vingt millions d'habitants sur un territoire compact, « leur science, leur talent d'organisation, leur grand patriotisme, leurs qualités militaires et économiques, leur natalité croissante sont des facteurs dont aucune défaite, aucune humiliation, aucun traité de paix ne pourra les priver et qui seront en contradiction criante avec la situation nouvelle qu' on veut leur imposer. " Même morcelée, même si son unité est supprimée en droit public, "rien ne pourra empêcher que son passé glorieux, le souvenir des vertus admirables qui se manifestent dans la guerre actuelles, ne maintiennent l'unité des âmes en dépit de tous les artifices et de toutes les restrictions. » Andrássy précise aussi que l'esprit militariste ne pourra être supprimé d'un trait. Les Allemands chercheront leur salut dans leur armée qui pourra leur garantir de nouveau « une place au soleil $»{ }^{22}$ Et pour illustrer cela, il se tourne vers l'Histoire et décrit l'échec de Napoléon dans sa tentative d'interdire à la Prusse d'avoir une grande armée : " la Prusse inculqua à son peuple une discipline nouvelle, et au moment où la lutte recommença, sa force se révéla plus grande qu'auparavant. »

Connaissant bien sa Hongrie natale, Andrássy explique que « les Hongrois n'ont accepté ni la domination turque, ni l'absolutisme autrichien - contre lequel son père s'est battu et fut même pendu en effigie pour avoir pris les armes en 1848 - : ils ne s'inclineront pas non plus devant les nouveaux dangers, ils ne 
laisseront briser ni leur courage ni leur conscience nationale. $»^{23}$ Il affirme aussi que les bulgares et surtout les turcs ne se laisseront pas faire non plus, avant de conclure qu'un arrangement grâce auquel tous les pays puissent garder leur dignité serait un meilleur garant pour la paix que la destruction de l'adversaire.

Même si les prévisions de Jules Andrássy sur la domination russe et anglaise se sont révélées plutôt fausse, il faut reconnaître que le Hongrois a réussi à saisir les enjeux de l'Europe d'après-guerre avec une logique toute « bainvillienne » (celle qui va chercher dans l'histoire les dénouements de l'avenir). C'est en effet l'historien et journaliste Jacques Bainville qui allait mener à l'excellence cette science de la prospective non quantitative en s'appuyant sur ses connaissances immenses de l'histoire et de la géographie : dans ses Conséquences politiques de la paix, livre publié en 1920 en réponse aux Conséquences économiques de la paix de Keynes, il allait annoncer presque tout ce qui allait se passer dans les vingt ans à venir, y compris en Hongrie. Mais sa vision serait basée sur le partage de l'Europe entre l'Allemagne et la Russie, au lieu de l'Angleterre et de la Russie comme l'avait prédit Andrássy. Ce dernier, tout de même, avait eu le mérite d'avoir annoncé, bien avant sa défaite que l'Allemagne allait se relever même si l'on le terrassait après la victoire.

Le premier article publié par Andrássy dans la Revue après la guerre, intitulé « La Hongrie et la Paix », était tout comme les précédents principalement destiné aux lecteurs français. Il n'y raisonne plus sur la nécessité d'arrêter la guerre, mais sur celle d'éviter qu'elle ne recommence en Europe orientale dans un conflit entre « les races et les partis ». Éviter, ensuite, que la région devienne le foyer de l'anarchie et de la révolution, et, enfin, faire accepter la victoire à tous les peuples de l'Europe orientale, y compris aux Hongrois. ${ }^{24}$

Il explique que la Hongrie ne cherche pas à s'opposer aux Traités de Paix et accepte le nouvel ordre des vainqueurs. Selon lui, la Hongrie se réjouit du contrôle anglais sur les Détroits « dont la défense contre toute convoitise moscovite » est dans l'intérêt de son pays. Toutefois, elle risque de tomber dans l'anarchie si elle est dépecée ou de succomber au danger bolchevique. Andrássy poursuit son analyse en affirmant que si la Hongrie est contentée, elle travaillera pour réconcilier les deux camps, tandis qu'une Hongrie lésée se jettera dans le bras du pangermanisme. Il tend la main vers la France, bien qu'il ne comprenne pas pourquoi elle s'acharne à détruire la Hongrie. Enfin, il assure que la Hongrie ne s'opposera désormais plus à la politique de la France et que si cela est arrivé dans le passé, c'était par la faute du système des alliances. ${ }^{25}$

Jules Andrássy annonçait nombre d'évènements à venir : en mars 1919, la République des Conseils était proclamée à Budapest; en 1927, la Hongrie se liait au premier groupe des mécontents des Traités de Paix chapeauté par l'Italie de Mussolini avant de retomber, malgré elle, dans l'itinéraire forcé de l'alliance avec l'Allemagne après l'Anschluss dix ans plus tard. Dans son article de novembre 
1916 comme dans celui de janvier 1919, Andrássy prévoyait le relèvement de l'Allemagne et le retour de la Hongrie dans son giron, presque deux décennies avant les faits, et en faisait l'annonce avant Bainville.

Poursuivons l'analyse de l'article de janvier 1919. Andrássy affirmait que le choix de l'Entente entre la lutte contre la Hongrie, qui lui résistait, et la trahison envers les petits alliés était un faux problème. Il proposait plutôt que l'Entente trouvât une manière de respecter les petits alliés tout en maintenant la Hongrie dans son intégrité, qui les respecterait à son tour. Il passe, un après l'autre, les états successeurs au crible de sa logique géopolitique.

La Tchécoslovaquie ne sera pas viable, écrit-il, car elle est multiethnique, étranglée de deux côtés par sa trop grande population allemande de Silésie et de Moravie qui lui donne une forme de guêpe. L'étroite bande de terre unissant la Bohême à la Slovaquie rendra le nouvel Etat impossible à défendre. De plus, si la Tchécoslovaquie voulait respecter la politique des nationalités, elle devrait renoncer à la partie la plus riche de la Bohême à l'avantage des populations allemandes. Selon Andrássy, l'impérialisme économique était à l'origine de la motivation des leaders tchèques; de la libération des frères on était passé à une guerre de conquête.

En ce qui concerne les Slaves du sud, Andrássy critique l'accroissement territorial de la Serbie déjà très bigarrée aux dépens de la Hongrie, par une région de 4 millions d'habitants dont seulement 700000 sont Serbes, les autres étant hongrois, allemands ou roumains.

On peut dire, en revanche, qu'Andrássy s'est trompé sur l'avenir proche de la Roumanie, car il prévoyait qu'elle aurait des soucis insurmontables en raison de ses fortes minorités hongroise et saxonne en Transylvanie. Mais ses prédictions sur les problèmes encourus avec la Bulgarie, sur la question de la Dobroudja, et avec la Russie - la Roumanie venait de prendre possession de la Bessarabie - se sont révélés exacts et se sont effectivement matérialisés respectivement au lendemain de la Seconde Guerre mondiale et après la Guerre froide (avec la création de la Transnistrie sous protectorat russe). Andrássy fondait toutes ses observations sur le fait que les pays, en se gonflant d'éléments étrangers, allaient produire de nouveaux irrédentismes et mettraient en danger la région. ${ }^{26}$

Le deuxième article d'Andrássy est rédigé en anglais, car il était destinée aux anglo-saxons et plus particulièrement aux américains résidant à Paris pendant les conférences de paix. Le Hongrois y fait l'éloge de Wilson « qui sous l'influence du génie de sa nation ${ }^{27}$ a été capable de résoudre le problème de l'autodétermination des nationalités en le subordonnant à l'intérêt général. Toutefois, pour Andrássy, les conditions préalables de ce programme sont le maintien de l'intégrité du territoire de la Hongrie, afin que les Hongrois ne soient pas obligés de lutter pour leur existence. Cet objectif n'est pas selon lui de l'expression d'intérêts privés subjuguant l'intérêt commun, mais, au contraire, celle d'un effort 
en vue de faire prospérer l'intérêt général. Dès lors, il faut maintenir la Hongrie millénaire en y créant des autonomies pour les différentes minorités ; solution qui ne mettrait pas en danger les pays voisins de la Hongrie. Mais cela implique de faire une exception aux principes de Wilson en contestant l'impérialisme des Tchèques, des Roumains et des Serbes. ${ }^{28}$ Bien que défendable du point de vue de la Hongrie, l'argument avait bien peu de chance de convaincre les grandes puissances victorieuses, compte tenu des promesses faites pendant la Grande Guerre à chacun des groupes ethniques ou États successeurs - bientôt rassemblés sous l'appellation de « Petite Entente » - et à l'Italie.

Dans ses deux articles de 1919, Andrássy montre qu'il était prêt à considérer l'attribution de larges autonomies aux minorités de la Hongrie, dans l'esprit de la déclaration de confédéralisation de l'Empire faite par l'empereur Charles le 16 octobre $1918 .{ }^{29}$ L'introduction d'autonomies auraient été un premier pas vers une décentralisation du royaume de Hongrie jusqu'alors très unitaire : « si au lieu de créer des irrédentismes nouveaux, on se contente d'une autonomie complète pour toutes les races du pays $\gg .{ }^{30}$ La solution qu'il préconisait était finalement la fédéralisation de la Hongrie, en accordant des autonomies aux différentes nationalités du royaume, et la confédéralisation de l'Europe centrale sous la forme d'une alliance essentiellement économique entre les états successeurs de l'Autriche-Hongrie. Les propositions faites dans ses articles de la Revue allaient très loin, aussi loin qu'il pouvait aller. Au pays, à Budapest, il devait taire ce projet fédéraliste qui n'était guère populaire et, au fond, Andrássy ne pouvait imaginer une Hongrie séparée de ces régions d'avant 1918, dont l'indépendance avait été chèrement payée par son père, qui rappelons-le, avait été pendu en effigie après 1848. Aurait-il fallu arriver à l'évidence que les petites concessions proposées ne pourraient ni satisfaire les états successeurs ni convaincre l'Entente? Dans ces articles de 1919, il espère encore arriver à dénouement différent.

\section{Conclusion}

Suite aux dispositions extrêmement sévères des Traités de Paix - celui de Trianon pour les Hongrois - la Hongrie allait effectivement être poussée dans les bras de l'Allemagne. Dans ses réflexions prospectives, Andrássy annonçait déjà les thèses de Jacques Bainville qui allait arriver à des conclusions similaires dans ses Conséquences politiques de la paix, ouvrage dans lequel ce dernier devait qualifier de " trajectoire forcée ${ }^{31}$ celle de la Hongrie se rapprochant de l'Allemagne afin de trouver un soutien pour obtenir des réparations territoriales.

Il est indéniable que les écrits d'Andrássy montrent une certaine sympathie envers les anglo-saxons. Il avait eu des contacts avec les Anglais pendant la guerre et avait reçu des signes d'encouragements pour sa politique de sortie de guerre de 
la part de Lord Lansdowne (ancien ministre des Affaires étrangères de Sa Majesté). ${ }^{32}$ Andrássy portait une grande admiration pour le Président Wilson et les États-Unis. Il avait, en revanche, moins de considération pour la France, dont il critiquait la politique en Europe centrale. Cependant les efforts qu'il déploya en Suisse, avec l'aide de Félix Vályi, montrent qu'il ne perdait pas l'espoir de contribuer à la modification la politique de la France dans la région. Du reste, son engagement ne fut pas vain, car, à partir de 1920, la France du gouvernement Millerand commença à s'intéresser à une confédération danubienne centrée à $\mathrm{Bu}$ dapest, en reconnaissant que la multiplication des états successeurs avaient affaibli la région en la rendant plus exposée à l'impérialisme allemand et soviétique. Jules Andrássy, connaissant la carte et l'histoire de sa région, avait été l'un des premiers à tirer la sonnette d'alarme, en langue française, et à stigmatiser la mauvaise paix qui était en train d'être entérinée par l'Entente, en ce qu'elle allait ouvrir deux décennie dites du système de Versailles qui allaient finir tragiquement, comme on le sait.

En ce qui concerne Félix Vályi, ses activités pour la cause légitimiste hongroise et ses contacts avec la France, ses diplomates et ses hommes du renseignement (Haguenin et François-Poncet), révèlent qu'il avait le rôle de rapprocher la France de l'Autriche-Hongrie puis de la Hongrie. Sa revue contribua à alimenter un débat large impliquant des personnalités de gauche comme de droite et à faire connaître les avis de la Hongrie sur la guerre comme sur la paix. Dans le cours de nos recherches, nous n'avons trouvé aucune documentation qui puisse expliquer l'interruption de la publication en 1920. Mais la fin de la revue semble montrer que cette dernière avait pour double objectif d'encourager les belligérants à terminer la guerre au plus vite et à réaliser une paix rapide et juste. Elle échoua tout aussi bien dans son ambition de favoriser une fin de guerre plus rapide que dans sa mission d'empêcher la signature des Traités de Paix tels qu'ils se présentaient, considéré par les vaincus comme des diktats destructeurs. Son existence n'avait donc plus lieu d'être.

Notes

1 La Séparation des Églises et de l'État vue par les journaux étrangers à partir des notes du Service de la Sûreté Générale du Ministère de l'Intérieur, réunies et présentées par Maurice Gelbard, 2005, http://theses.ulb.ac.be/ETD-db/collection/available/ULBetd-08272007103325/; Henri Montety (de), « Clemenceau et le Magyar Szó », Hungarian Studies, vol. 25, juin 2011, Budapest, Akadémia Kiadó, p. 143.

2 «Introduction », Revue politique internationale, vol. 1, février, 1914, p. 5.

$3 \mathrm{http}: / / \mathrm{www}$.academie-francaise.fr/les-immortels/andre-francois-poncet (consulté le 19 mars 2013). 
4 «Avis au lecteurs » attaché au premier numéro publié à Lausanne, $\mathrm{n}^{\circ}$ 9-10, septembre-octobre 1914.

5 József Kardos, « Ifjabb Andrássy Gyula Gróf utolsó évei » [Les dernières années du comte Jules Andrássy le jeune], Történelmi Szemle, XXXVI, 3-4, 1994, pp. 299-300.

6 József Kardos, Legitimizmus : Legitimista politikusok Magyarországon a két világháború között [Les politiciens légitimistes en Hongrie entre les deux guerres], Budapest, Korona Kiadó, 1998, pp. 11-12.

7 György Szmrecsányi, du Parti du Peuple, et le Marquis György Pallavicini, du Parti de la Constitution, faisaient parti de ce groupe de légitimistes à l'intérieur de l'ABC. Ignác Romsics, István Bethlen: A Great Conservative Statesman of Hungary, 1874-1946, Social Science Monographs, Boulder, Colorado, 1995, p. 99.

8 Raphael Patai, The Jews of Hungary: History, Culture, Psychology, Wayne State Univ. Press, 1996, p. 382.

9 Romsics, op. cit., p. 82.

10 ADMAE (Archives diplomatiques du Ministère des Affaires étrangères), Z Europe 1918-1929, Hongrie (Z Europe, H.), Propagande 65/1. Berne, 25 mars 1919.

11 Az európai és a nemzetközi integráció jelentősége ifj. gr. Andrássy Gyula eszmerendszerében [La signification de l'intégration européenne et internationale dans la pensée du comte Jules Andrássy le jeune], http://ifjgrofAndrássygyula.hu/?page_id=107 (consulté le 20 mars 2013); «Émile Haguenin (né en 1872) : Ancien élève de l’École normale (promotion 1893), il est catholique mais se dit proche, par ses idées, du Parti socialiste ; il prend fait et cause pour Alfred Dreyfus. Devenu professeur d'allemand à l'École normale. Avant la guerre, il enseigne à l'université de Berlin. Rentré en France à l'éclatement des hostilités, il accepte fin 1915 la proposition de Philippe Berthelot de partir à l'ambassade de France à Berne comme « Directeur du Bureau de presse ». Agent d'influence et de renseignement, propagandiste, Haguenin est en correspondance suivie pendant la Grande Guerre avec le Quai d'Orsay et les services de renseignements français. En décembre 1916, il transmet à Briand - dont il est proche - l'offre de médiation en faveur de la paix de von (Harry) Kessler, ainsi que d'autres propositions, émanant d'émissaires austro-hongrois non officiels (socialistes). À compter de février 1919, Haguenin est donc nommé « chef de la mission d'information française à Berlin ». Dans le cadre de l'édification d'un nouveau système de sécurité collective en Europe, il va certes œuvrer pour un rapprochement franco-allemand, mais par le truchement d'une entente entre industriels des charbonnages et de l'acier des deux pays. » Lahaie, Olivier, «Face à l'Allemagne vaincue, les services de renseignement français (novembre 1918-avril 1919) », Revue Historique des Armées, 251, 2008, note 57.

12 ADMAE, Z Europe 1918-1929, Hongrie, Propagande 65/1. Berne, 25 Mars 1919. D'autres sources prétendent que c'est un certain Rodolph Kremmer de United Press qui en était le directeur. MOL, K64, 3 cs., 1921-1-177, Budapest, 17 avril 1921, Gen. Berzeviczky à Khun-Hederváry du dép. politique et à l'Amb. Kánya.

13 PRO (Public Record Office, Londres), FO (Foreign office) 371 3529, 36671, Rumbold to Curzon, 1. March 1919.

14 Windischgraetz, Ein Kaiser kämpft für die Freiheit, Verlag Herold, Wien, 1957, pp. 137-138.

15 Ibid.

16 Henri Montety (de), op. cit., p. 3.

17 Jules Andrássy, «Le problème de la Paix », Revue politique internationale, no 23-24, déc. 1916.

$18 \quad$ Ibid. pp. 101-104. 
19 Landry Charrier, « À la recherche d'une paix de compromis : Kessler, Haguenin et la diplomatie officieuse de l'hiver 1916-1917 », Histoire@Politique.Politique, culture, société, №11, mai-août 2010, www.histoire-politique.fr (consulté le 21 mars 2013).

20 Traditionnellement : Angleterre, France, Russie, Japon. Ici, Andrássy semble insister sur l'Italie, comme le quatrième Etat.

21 Andrássy, « Le problème... », p. 107.

22 Ibid. pp. 107-108.

23 Ibid. p. 108

24 Jules Andrássy, « La Hongrie et la Paix », Revue politique internationale, no 36, jan-fév. 1919, pp. 3-4.

$25 \quad$ Ibid. p. 5.

26 Ibid. pp. 6-10.

27 Jules Andrássy, « National Self-Determination », Revue politique internationale, $\mathrm{n}^{\circ} 37-38$, mai-juin 1919, pp. 183, 185.

28 Ibid. 186.

29 Cette déclaration n'impliquait que l'Empire d'Autriche et pas le Royaume de Hongrie.

$30 \quad$ Ibid. p. 20

31 Bainville, op. cit., Paris, 1920, pp. 112-113.

32 http://ifjgrofAndrássygyula.hu/?page_id=107

\section{The Secret Exploits and Journalism of Count Gyula Andrássy the Younger in Switzerland and His Visions of the Post-War Era}

\section{Summary}

Having fled Hungary to spend eight months in Switzerland as an exile, Gyula Andrássy sought out counter-revolutionary organs of the media as well as the journalist Félix Vályi in order to promote, through propaganda, the fall of communism in Hungary, the return of the king from exile and the fight for the territorial integrity of Hungary. During his time in Switzerland, Andrássy wrote two articles in the Revue politique internationale, headed by Vályi in Lausanne. His articles shed light on his post-war ideas regarding the future of Central Europe and Hungary. He hoped to exert an influence in the politics of the victorious states in the region.

Keywords: Gyula Andrássy, Félix Vályi, Switzerland, France, legitimism, Great Britain, Entente, Quadruple Entente, intelligence, confederation, Anti-Bolshevik League 